\section{Fatores de proteção relacionados à promoção de resiliência em pessoas que vivem com HIV/AIDS}

\author{
Protective factors and resilience in people \\ living with HIV/AIDS
}

\author{
I Instituto de Psicologia, \\ Universidade Federal do Rio \\ Grande do Sul, Porto Alegre, \\ Brasil. \\ 2 Grupo de Interação \\ Social, Desenvolvimento e \\ Psicopatologia, Porto Alegre, \\ Brasil. \\ 3 Centro de Estudos \\ Psicológicos sobre Meninos e \\ Meninas em Situação de Rua \\ Porto Alegre, Brasil. \\ Correspondência \\ F. T. Carvalho \\ Instituto de Psicologia, \\ Universidade Federal do \\ Rio Grande do Sul. \\ Rua Ramiro Barcelos \\ 2600/111, Porto Alegre, RS \\ 90035-006, Brasil. \\ torresdecarvalho@yahoo.com.br
}

\begin{abstract}
The aim of this theoretical review was to articulate the resilience concept with key aspects in the lives of people living with HIVIAIDS. We emphasize the analysis of protective factors traditionally related to resilience (personal characteristics and social and affective support networks). The reviewed studies show important protective factors that contribute to the health and well-being of people with HIVIAIDS, such as cognitive coping and acceptance of their HIV status, family participation in treatment and family support, the role of governmental and nongovernmental institutions, and religious beliefs. The concept of resilience defined as a dynamic process that allows human beings to overcome adversities is essential for understanding HIV infection and treatment of AIDS patients. It helps decrease stigmatization and prejudice towards the disease and patients. It also helps alter the notion that living with AIDS is incompatible with well-being and quality of life and fosters the creation of new HIVIAIDS prevention and treatment perspectives.
\end{abstract}

HIV; Acquired Immunodeficiency Syndrome; Review Literature
Fernanda Torres de Carvalho 1,2 Normanda Araujo de Morais 1,3 Sílvia Helena Koller 1,3

Cesar Augusto Piccinini 1,2
O HIV/AIDS (vírus da imunodeficiência humana/ síndrome da imunodeficiência adquirida) é uma infecção que atingiu o estágio de pandemia no mundo 1, apresentando-se no Brasil como uma epidemia de proporções preocupantes 2 . Desde o seu surgimento, na década de 1980, vêm-se observando os fenômenos de heterossexualização, feminização, interiorização e pauperização da epidemia 3,4, o que demonstra que a infecção não está mais restrita ao que se considerava como grupos de risco (homossexuais, usuários de drogas injetáveis e profissionais do sexo, por exemplo), atingindo a população de forma geral 5,6.

Concomitante à disseminação do HIV/AIDS na população ao longo dos anos, novas opções de tratamento foram sendo desenvolvidas, o que aumentou a sobrevida e a melhora da qualidade de vida dos atingidos 7,8. Nesse contexto, se a preocupação no início da epidemia era a iminência de morte logo após o diagnóstico, atualmente, soma-se ao medo da morte a necessidade de reestruturação da vida, a fim de conviver com a presença do vírus no organismo. Os avanços no diagnóstico e tratamento do HIV/AIDS vêm tornando essa infecção cada vez mais semelhante às doenças definidas como crônicas 9 , e, conforme Siegel \& Lekas ${ }^{9}$, esses avanços geraram um impacto considerável na vida dos portadores de HIV/AIDS, diminuindo o temor sobre a iminência da morte e possibilitando a permanência de relações sociais, de trabalho, de lazer e afetivas na vida. 
Pesquisas exaustivas vêm demonstrando o impacto negativo do vírus sobre a vida das pessoas, contribuindo, assim, para o avanço dos conhecimentos nessa área. No entanto, igual preocupação não tem sido dada à investigação dos fatores de proteção no desenvolvimento dos pacientes com HIV/AIDS, os quais podem estar contribuindo para a saúde, bem-estar, qualidade de vida e resiliência desses indivíduos.

Desde o momento da infecção até o surgimento de algum sintoma, podem transcorrer meses ou anos, sendo esse intervalo difícil de definir. O tempo médio de desenvolvimento de sintomas tem sido de cinco a oito anos, porém existem pessoas infectadas há 15 anos ou mais que nunca tiveram complicações de saúde por causa do HIV/AIDS. O que existe de diferente nessas pessoas? Que possíveis fatores de proteção podem estar envolvidos? Sabe-se que questões como a adesão ao tratamento ou a presença de subtipos virais resistentes a drogas são importantes nesse processo. Porém, resta a necessidade de se conhecerem os aspectos psicossociais envolvidos, tanto na adesão ao tratamento, como na aquisição de comportamentos que podem contribuir para aumentar as resistências do organismo, retardando a manifestação da doença.

$\mathrm{Na}$ área da psicologia, a investigação sobre os fatores de proteção e resiliência têm sido feitas no estudo do desenvolvimento humano, particularmente com populações consideradas sob risco. Nesses casos, busca-se compreender o que está envolvido quando situações de adversidade são vivenciadas e superadas pelos indivíduos. Esse campo teórico desenvolveu o conceito de resiliência como o potencial saudável que alguns indivíduos apresentam em situações de possibilidade de doença. Poderia, então, a resiliência dos portadores de HIV/AIDS contribuir para sua adesão ao tratamento e qualidade de vida?

Visando a uma maior compreensão dessas questões, o presente artigo busca, a partir da revisão da literatura, articular o conceito de resiliência com questões da realidade de vida de pessoas portadoras de HIV/AIDS. Em especial, será enfatizada a análise dos fatores de proteção tradicionalmente mencionados na literatura como promotores de resiliência (características individuais e rede de apoio social e afetiva), a partir da devida articulação e contextualização com os fatores de risco vivenciados por pessoas portadoras de HIV ou com AIDS.

Para a realização deste artigo, foi feita uma revisão exaustiva nas principais bases de dados (PsycInfo, MEDLINE, SciELO, Biblioteca Virtual de Saúde, BIREME), utilizando-se inicialmente os termos resiliência (resilience) e HIV/AIDS (HIV/SIDA), sem restrição à data de publicação dos estudos. Em um segundo momento, foram acrescentados os termos fatores de proteção (protective factors) e fatores de risco psicossociais (psychosocial factors), tendo em vista que alguns artigos encontrados mencionavam o termo resiliência sem aprofundar o conceito ou sem que este fizesse parte dos objetivos do trabalho. Por fim, foram acrescentados os termos apoio social (social support), apoio familiar (family support) e aspectos sociais (social issues), mantendo-se constante o termo HIV/AIDS.

Por intermédio deste procedimento, reuniuse um conjunto de 68 artigos e capítulos de livros nacionais e internacionais. Para fins de análise desse material, utilizaram-se os pressupostos de Masten \& Garmezy 10, no que se refere aos fatores promotores de resiliência (atributos disposicionais das pessoas, laços afetivos no contexto familiar e outros contextos e sistemas de suporte social). Foram, então, feitas leituras e discussão sistemática de todos os textos disponíveis, com destaque para os principais achados e evidências que corroborassem as categorias propostas por Masten \& Garmezy, a fim de identificar potenciais fatores de proteção para pessoas que vivem com HIV/AIDS. Dessa forma, o que antes aparecia de forma isolada em outros textos, neste artigo é reunido e articulado sob a perspectiva de fatores de proteção que podem contribuir para a promoção de resiliência.

A seguir, serão inicialmente introduzidos os conceitos de resiliência e de fatores de risco e proteção para que, em seguida, sejam apresentadas e discutidas as evidências derivadas da literatura sobre as categorias de Masten \& Garmezy 10.

\section{Resiliência}

Há pouco mais de vinte anos, o tema da resiliência vem se constituindo como uma área de interesse e pesquisa na psicologia. A partir do final da década de 1990, sobretudo, as publicações sobre esse tema ganharam mais ênfase por parte dos pesquisadores da psicologia, principalmente daqueles que trabalham com populações em situações de risco (vítimas de violência, crianças e adolescentes em situação de rua, entre outros). A ênfase desses estudos, por sua vez, esteve muito centrada no desenvolvimento de crianças e adolescentes, tendência que continua ainda hoje, embora com menor intensidade.

Inicialmente, o estudo da resiliência foi proposto pela física e sua definição estava relacionada à capacidade de um material recobrar o seu estado anterior, depois de ter passado por alguma deformação 11,12. Ao ser incorporada pela ciência psicológica, a resiliência passou a ser 
vista como o conceito que explicaria o porquê de, mesmo em situações de adversidades sociais, econômicas e psicológicas, alguns indivíduos conseguirem sobreviver e alcançar o bem-estar em suas vidas, enquanto outros não o obtinham 10,13,14,15,16,17.

A princípio e, principalmente, em virtude da transposição desse conceito da física para a psicologia, a definição do termo resiliência esteve muito relacionada à idéia de "invulnerabilidade" (resistência absoluta ao estresse) e de "adaptação". No entanto, essas concepções têm sido fortemente criticadas por causa do seu caráter estático e absoluto, optando-se por uma visão mais dialética e processual da resiliência 11,12. De acordo com esta perspectiva, mais do que recobrar um estado anterior após uma situação de estresse/trauma, a resiliência, no contexto humano, implica "superação" da dificuldade enfrentada, possibilitando uma re-significação e/ou a construção de novos caminhos diante da adversidade. Trata-se, então, de uma capacidade a ser construída ao longo do processo de desenvolvimento humano. Portanto, de acordo com Junqueira \& Deslandes 18 (p. 233), resiliência é entendida como uma "reafirmação da capacidade humana de superar adversidades e situações potencialmente traumáticas".

Além disso, resiliência não é uma característica estática, mas um processo, que não é ativado para toda e qualquer situação, nem a todo momento, como por vezes aparecia na idéia original associada à invulnerabilidade. Assim, entende-se que a resiliência não é um processo linear nem estanque, visto que um indivíduo pode se apresentar como resiliente diante de uma situação e, posteriormente, não o ser diante de outra situação e, eventualmente, até mesmo diante da mesma situação 19. Com isso, entende-se que resiliência não implica atingir um estado de bemestar constante. O entrejogo das variáveis individuais e contextuais configurará constantemente a maior ou menor capacidade de cada pessoa, em momentos específicos, de lidar de forma positiva com a adversidade, não sucumbindo a ela 16 .

As bases da resiliência são tanto constitucionais quanto ambientais, não havendo, por conseguinte, uma quantidade fixa, mas, sim, que varia de acordo com as circunstâncias. A resiliência é entendida, portanto, não somente como uma característica do indivíduo, como uma capacidade inata, herdada por alguns "privilegiados", mas também como parte da interação dinâmica existente entre as características individuais e a complexidade do contexto social 12,18,20,21,22.

\section{Fatores de risco e proteção}

A definição de resiliência, a partir da compreensão da interação do indivíduo com o seu ambiente, implica o entendimento dinâmico dos chamados fatores de risco e proteção. Os fatores de risco são os eventos de vida que, quando presentes, aumentam a probabilidade de o indivíduo apresentar problemas físicos, psicológicos e sociais. Já os fatores de proteção referem-se às influências que modificam, melhoram ou alteram respostas pessoais a determinados riscos de desadaptação 12,23.

Tanto no conceito de fatores de risco, quanto no de proteção, a literatura tem destacado a necessidade de se enfatizar uma abordagem de "processos". Ou seja, a priori, nenhuma variável é fator de risco ou proteção, uma vez que qualquer fator pode, potencialmente, ser de risco em uma situação e protetor em outra, dependendo justamente da relação estabelecida entre as variáveis individuais e o contexto sócio-ambiental 12,23. Dessa forma, as pesquisas sobre resiliência se voltam ao estudo dos mecanismos específicos através dos quais determinadas variáveis produziram resultados particulares 15,17. Além disso, os fatores de risco e proteção serão assim considerados conforme a percepção de cada indivíduo ${ }^{19}$. Isso porque um fator pode ser considerado como "de risco" em um contexto e situação específica, enquanto em outro contexto, ou mesmo, em um momento diferente no tempo, ser considerado "de proteção".

Sobre os fatores de risco, é importante destacar que a resiliência aparece em situações que expõem ao risco. Portanto, sem "risco, adversidade ou situação estressora" não se pode falar em resiliência. De acordo com Cowan et al. 24, haveria três formas pelas quais os indivíduos podem superar a situação de risco: (i) eles podem não ter vivenciado muitas das experiências estressantes que tendem a ser associadas com o risco; (ii) eles podem desenvolver habilidades de enfrentamento para contra-atacar o risco e responder com mudanças que cancelam o impacto negativo do risco e o fazem avançar a novos níveis de adaptação e (iii) eles podem dispor de algum fator de proteção que diminua o impacto do risco.

O presente artigo destaca, em especial, esse último ponto, ao buscar compreender os possíveis fatores de proteção no enfrentamento da infecção pelo HIV/AIDS. De fato, a literatura psicológica tem sido fortemente marcada pelo estudo do "risco" e suas conseqüências negativas ao desenvolvimento humano 19,25. Dessa forma, é mais comum a existência de estudos sobre os eventos negativos que aumentam a probabilidade de resultados negativos da infecção pelo HIV/AIDS 
na vida das pessoas (abandono do tratamento, violência doméstica em decorrência de revelação de diagnóstico, preconceito, estigmatização, abuso de drogas, por exemplo) do que o foco sobre fatores de proteção, cuja presença pode estar contribuindo para o desenvolvimento de respostas positivas por parte dos indivíduos.

Contudo, a partir do movimento da denominada Psicologia Positiva, sobretudo a partir do ano 2000, uma nova preocupação tem sido trazida aos estudos da psicologia e, particularmente, da resiliência 19,22,26,27,28,29. Trata-se de compreender os fatores e processos que promovem $o$ desenvolvimento psicológico sadio e quais os aspectos que são responsáveis por fortalecer e construir competências nos indivíduos. Essa nova perspectiva, como se vê, encoraja o foco nos estudos sobre os fatores de proteção, sobretudo para se desenvolverem estratégias de prevenção mais eficazes.

Essas estratégias, de acordo com Robinson 30 , deveriam consistir tanto na evitação do risco, como na promoção de um funcionamento psicológico mais saudável após a exposição ao mesmo. Nesse sentido, além de ser protetor ao indivíduo evitar situações que o exponham ao risco, poderia ser também protetora a experiência do risco e a reconstrução psicológica que tal experiência proporcionasse. Essa concepção parece refletir a realidade de alguns indivíduos portadores de HIV/AIDS, como é o caso de homens que relatam terem passado a levar uma vida mais saudável, após o diagnóstico; ou de mulheres que estavam afastadas de tratamento e que o retomaram em virtude de uma gravidez 31 . É como se a soropositividade, sendo uma experiência extremamente marcante, mesmo que potencialmente adversa, gerasse em alguns casos a transformação dos hábitos de vida. Cuidados com a saúde (física, mental e espiritual) passaram a fazer parte da rotina de pessoas que, antes do diagnóstico de HIV/AIDS, informaram não ter esse tipo de preocupação.

Ainda baseada no modelo da doença, a psicologia tem muito a avançar nos estudos e pesquisas sobre prevenção 28 . Diante disso, Seligman \& Csikszentmihalyi 28 defendem uma mudança de paradigma, de modo que a prevenção viesse de uma perspectiva focada na construção de competências e não na correção de fraquezas ou fragilidades. A necessidade de se enfatizar o estudo dos fatores de proteção sustenta-se, também, na afirmação de Cowan et al. 24, segundo a qual não é interessante relacionar eventos de vida estressantes a resultados adversos físicos e psicológicos, porque "os fatores de risco ocorrem muito mais freqüentemente que os resultados disfuncionais" 24 (p. 7). Em adição, na vida real, as pessoas movem-se constantemente "para dentro e para fora" de situações de risco, criando, assim, diferentes estados de adaptação psicológica. Nesse processo de busca de adaptação, por sua vez, os chamados fatores de proteção exercem um papel fundamental, uma vez que são eles os responsáveis por diminuir a probabilidade de um resultado negativo ou indesejável acontecer diante da presença do risco.

De acordo com Eckenrode \& Gore 32 , os fatores de proteção podem ser classificados em dois grupos: (i) pessoais e (ii) recursos do ambiente. Masten \& Garmezy 10, pioneiros no estudo da resiliência, por sua vez, subdividem os recursos do ambiente em dois subgrupos, dando especial destaque ao papel da rede de apoio social e também afetiva das pessoas para a promoção de resiliência. Dessa forma, identificaram três classes de fatores de proteção: (i) os atributos disposicionais das pessoas; (ii) os laços afetivos no sistema familiar e/ou em outros contextos que ofereçam suporte emocional em momentos de estresse e (iii) os sistemas de suporte social, seja na escola, no trabalho, nos centros religiosos, no serviço de saúde, que propiciem competência e determinação individual e um sistema de crenças para a vida. Verifica-se, portanto, que há semelhança entre definições de diversos autores, que abordam os fatores de proteção com base nas variáveis individuais/pessoais e ambientais. Todavia, a subdivisão proposta entre rede de apoio social e rede de apoio afetiva é bastante pertinente, pois a presença da primeira não implica necessariamente a segunda. Além do mais, dado o estigma vinculado à questão do HIV/AIDS na sociedade atual, ressalta-se a necessidade de haver maior clareza sobre o papel que as redes podem ter na vida dessas pessoas e na forma como estas lidam com a sua situação de infecção.

\section{Resiliência e HIV/AIDS}

Poucos são os estudos que relacionam resiliência e fatores de risco e proteção com a infecção pelo HIV/AIDS. Embora alguns estudos tratem de aspectos relacionados à promoção de resiliência (suporte social familiar, principalmente), a relação que é feita com a resiliência é bastante superficial e indireta 33,34. Ademais, termos como "adaptação psicológica" e "enfretamento" são mais freqüentes nesses estudos que o conceito de resiliência 35,36,37. Este último, por sua vez, é mencionado apenas tangencialmente, pouco definido e problematizado, não parecendo ser o foco principal dos artigos 33,34,35,36,37.

Uma possível justificativa para essa lacuna é que a infecção pelo HIV/AIDS é um fenômeno 
relativamente recente, assim como os estudos e pesquisas acerca da resiliência. Incorporado recentemente à psicologia, esse conceito precisa de um pouco mais de tempo para ser absorvido pelas ciências biológicas e da saúde. Mesmo na psicologia, área em que o conceito é mais conhecido, poucos são os colegas que se dedicam ao estudo de HIV/AIDS; conseqüentemente, a resiliência não tem sido investigada no contexto dessa infecção, no qual o enfoque é ainda predominantemente biológico e médico. Apresenta-se, a seguir, a revisão dos estudos sobre potenciais fatores de proteção relacionados à promoção de resiliência em pacientes portadores de HIV/AIDS, dividindo-os a partir da proposição de Masten \& Garmezy 10, a saber: atributos disposicionais das pessoas, laços afetivos no contexto familiar e outros contextos e sistemas de suporte social.

\section{Atributos disposicionais das pessoas}

A primeira classe de fatores de proteção promotores de resiliência diz respeito aos atributos pessoais 10. De maneira geral, estudos sobre resiliência em diversas populações têm mostrado que uma pessoa desencadeia um processo de resiliência quando apresenta senso de auto-eficácia, senso de humor, força para responsabilizar-se por outros ou ser parte de um esforço comunitário mais amplo, além de possuir capacidades intelectuais, como, por exemplo, o potencial para insight 38 . Masten \& Garmezy 10 também mencionam fatores como o nível de atividade e sociabilidade da pessoa, a auto-estima e a autonomia. Tais características, porém, não implicam obrigatoriamente resiliência, uma vez que é preciso avaliar outras variáveis contextuais e os fatores de risco em cada situação. Além disso, como são características pessoais, apresentam-se sob diferentes formas de expressão para cada indivíduo.

Estudos com portadores de HIV/AIDS apresentam alguns fatores individuais que podem influenciar a saúde dessas pessoas. A análise de $\mathrm{Au}$ et al. ${ }^{39}$ sugeriu associação entre a saúde física e a psicológica dos pacientes, o que, para os autores, permite um entendimento integral da infecção. Ulla \& Remor 40 consideraram as variáveis psicológicas como co-fatores para a competência do sistema imunológico e, conseqüentemente, para a evolução do vírus no organismo. Esses estudos vêm indicando a influência do estresse, dos estilos de enfrentamento utilizados pelos indivíduos e dos hábitos de vida sobre o curso de desenvolvimento da AIDS.

De maneira geral, os estudos investigam os aspectos psicológicos e as interfaces com a saúde física a partir da avaliação das estratégias de enfrentamento (coping) da infecção, geralmente conceituadas como o conjunto de esforços realizado pelos indivíduos para lidar com demandas geradas por situações de estresse. Por exemplo, o enfrentamento cognitivo e a aceitação da realidade da infecção aparecem como um possível fator de proteção, na medida em que se associa a uma melhor qualidade de vida e a um menor sentimento de estresse 41. Para os autores, trata-se de um processo de aceitação ativa, que facilita a mobilização da pessoa em direção à tomada de atitudes em relação aos desafios encontrados. Isso é importante, porque estratégias que envolvem evitação ou negação da infecção são as descritas como mais associadas a sofrimento psicológico 42 e a baixos índices de qualidade de vida 41 .

O sentimento de auto-eficácia para adesão ao tratamento também tem sido descrito como um fator importante para a saúde dos portadores do HIV/AIDS. A crença na capacidade de aderir aos anti-retrovirais e a confiança nos seus benefícios estiveram associadas a melhor qualidade de vida e melhor saúde física de pacientes 43 . Em adição, os autores verificaram que indivíduos mais jovens e com maior escolarização tendiam a ter maior confiança nos benefícios da terapia anti-retroviral e sentiam-se mais capazes de aderir a ela.

Contudo, algumas pessoas não conseguem assimilar o resultado positivo para HIV/AIDS e podem precisar de um tempo para assumir uma postura mais ativa diante de sua infecção ${ }^{44}$. Sendo assim, podem-se identificar pessoas que negam o próprio resultado 45,46 , que duvidam da seriedade do teste diagnóstico 47 ou que têm dificuldade de tomar para si a responsabilidade da infecção ${ }^{48}$. Essa negação pode ser uma fuga da realidade, como uma tentativa do indivíduo de se adaptar a sua nova situação. Assim, enquanto ocorre esse processo, a pessoa deixa de buscar auxílio para sua saúde física e emocional, o que diminui a sua rede de apoio 41 . Um aspecto importante nessa reação inicial à infecção é o medo do estigma social da doença, que modifica e restringe a vida dos envolvidos 46 . Por todos esses motivos, a infecção tende a aumentar o estresse na vida dos indivíduos, estando a estratégia de negação associada à percepção da vida como um evento altamente estressante ${ }^{41}$.

Em conjunto, esses estudos mostram que, no contexto do HIV/AIDS, as diferenças individuais podem servir como fatores tanto de risco, quanto de proteção. A reação em face da soropositividade e as atitudes e comportamentos dos indivíduos a partir daí parecem se constituir em importantes fatores que precisam ser considerados para a promoção da saúde e da qualidade de vida desses pacientes. 


\section{Laços afetivos no sistema familiar ou outros contextos}

Estes fatores de proteção dizem respeito às relações afetivas dentro do sistema familiar ou que envolve outras pessoas 10. Encontra-se, nesse caso, o relacionamento familiar, entre amigos e a vida social em sua micro-estrutura. Trata-se de possíveis fontes de apoio emocional que um indivíduo pode acessar em situações de adversidade e que podem contribuir para que se estabeleça a resiliência. Muitos são os estudos que demonstram a importância desse fator na vida dos portadores de HIV/AIDS. A família e os amigos se constituem em expressivos fatores de proteção àqueles que precisam enfrentar a infecção. Diante disso, algumas considerações sobre a importância da família e dos amigos serão apresentadas a seguir, juntamente com considerações sobre o contexto microssocial dos indivíduos.

Já no recebimento do diagnóstico, o apoio familiar parece ser importante. Ao se imaginarem recebendo resultado positivo para HIV/AIDS, pacientes de uma clínica geral mencionaram que gostariam que alguém da família também fosse avisado 49 . Em alguns casos, a revelação do diagnóstico a familiares próximos esteve associada à maior percepção de apoio proveniente dessas relações 50 . Ademais, o apoio social vem sendo descrito como associado a menor sofrimento psicológico, menor freqüência de sintomas psiquiátricos 51 , além de a menores índices de ansiedade e depressão e melhor qualidade de vida 52. Mulheres que afirmaram receber apoio de suas famílias apresentaram menores índices de estresse e sentimento de solidão 53 . Ainda, a participação de familiares em grupos de apoio, mesmo que seja difícil para a família lidar com o diagnóstico de HIV/AIDS em um de seus membros, vem sendo identificada como algo positivo para o tratamento dos pacientes 52 .

Com o avanço do tratamento para AIDS, tornou-se extremamente importante que os pacientes tenham uma boa adesão aos esquemas terapêuticos, a fim de que estes sejam efetivos $7,54,55$. Essa adesão, entretanto, até o momento, não vem sendo sempre satisfatória 7 . Nesse contexto, o apoio social e familiar parece ser um fator importante, já que a adesão pode exigir a reorganização da vida diária e até a revelação da soropositividade às pessoas mais próximas 56 .

Considerando que a família é a principal fonte de apoio para soropositivos 47 , afastar-se desse contato pode ser prejudicial. O que ocorre em alguns casos é que o medo do preconceito e do isolamento faz com que os portadores evitem revelar o diagnóstico a familiares. Com isso, o enfrentamento da infecção torna-se um processo solitário. De uma forma geral, os indivíduos desenvolvem crenças de que, se revelarem seu diagnóstico, não serão mais aceitos pela família, que ficarão sós e abandonados 46 e que serão motivo de vergonha para seus familiares 46,57.

Além disso, a revelação a membros da família ou a amigos apresenta particularidades que vêm sendo descritas na literatura. Em um estudo com homens e mulheres soropositivos norte-americanos, os participantes referiram que os amigos para os quais revelaram o diagnóstico ofereceram maior apoio do que os familiares que foram informados 50 . Ainda, esses portadores tendiam a revelar mais a pessoas da família extensa do que à família nuclear. Considerando os familiares de grau mais próximo, os pais e irmãos do sexo masculino foram percebidos como pessoas que ofereciam menos apoio, o que fazia com que a participação ocorresse mais por parte das mães e das irmãs. Esses dados sugerem que as pessoas procuradas para a revelação sobre a soropositividade são aquelas identificadas como potencialmente apoiadoras, e que membros mais próximos da família podem não ser as principais escolhas.

Além da família e amigos, outra relação próxima e afetiva pode ser a do psicólogo-paciente. Turner 38 menciona o papel da terapia e da aliança terapêutica entre paciente e terapeuta como uma importante fonte de promoção de resiliência. Isso acontece em virtude de o terapeuta representar uma figura de confiança e apoio para o cliente. Em adição, o espaço da terapia favorece a afirmação da experiência trazida pelo paciente, sendo um espaço de descoberta de "recursos e capacidades" e de fortalecimento dessas "forças", por meio das características individuais e dos recursos externos. Junqueira \& Deslandes 18 ressaltam também o lugar de destaque exercido pelas relações microssociais em saúde. As autoras destacam esse espaço como promotores em potencial de resiliência, através do estabelecimento de vínculo e confiança entre os diferentes profissionais de saúde e os pacientes.

De forma geral, os estudos deixam clara a importância da família para a saúde de pessoas portadoras de HIV/AIDS. Em especial, o apoio de pessoas próximas parece ser um fator de proteção aos soropositivos, mas isso também pode ser estendido aos amigos e profissionais de saúde.

\section{Sistemas de suporte social}

Os sistemas de suporte fazem parte de um âmbito mais amplo da vida das pessoas 10 . Trata-se das relações estabelecidas com a escola, o trabalho, centros religiosos ou instituições de saúde. A fim de promoverem fatores de proteção aos indiví- 
duos, essas instâncias precisam propiciar a competência e a determinação individuais.

Alguns fatores têm sido descritos como de proteção aos indivíduos para a vida em sociedade. No contexto brasileiro, um desses fatores é a estrutura de centros de saúde disponível na rede pública. O Brasil tem sido um país modelo na luta contra a AIDS, com o oferecimento de tratamento gratuito na sua rede de saúde. Centros de referência no atendimento à infecção e à AIDS estão disponíveis aos pacientes, tanto para diagnóstico como para tratamento, com distribuição gratuita de medicamentos anti-retrovirais. Centros de pré-natal de referência têm conseguido diminuir a taxa de transmissão materno-infantil do HIV para índices inferiores a 3\% 58. Nos últimos anos, o Brasil ampliou a distribuição de medicamentos, prolongando a vida das pessoas, diminuindo as taxas de mortalidade 59. Mesmo que a situação não seja a ideal, o serviço prestado é de boa qualidade, sendo um modelo de atendimento internacionalmente reconhecido. Além do mais, a legislação brasileira prevê a proteção ao indivíduo portador do vírus, dando direito à privacidade quanto ao diagnóstico, proibindo a exigência de testes de HIV para admissão em empregos, bem como a demissão por causa da soropositividade. Muitos desses avanços na legislação são resultados de uma ação forte de organizações não-governamentais (ONGs) que, desde o início da epidemia, se mantêm ativas, no intuito de proteger a saúde e a cidadania dos portadores do HIV/AIDS. A partir da década de 1990, as diversas ONGs que surgiram voltadas a essa área trouxeram ações e um discurso de solidariedade, com crítica ao sentimento de pena e ao preconceito e estigmatização 60 .

Outro fator de proteção aos indivíduos portadores de HIV/AIDS pode ser a religiosidade, que tem se mostrado como um aspecto que pode ser intensificado a partir da vivência da soropositividade. Prado et al. 42 , entrevistando mulheres brasileiras, verificaram que o envolvimento religioso esteve relacionado a estratégias mais ativas de enfrentamento da infecção e a maiores índices de apoio social. Para os autores, o envolvimento religioso pode servir como um mecanismo de redução do processo de enfrentamento de evitação (negação) e, conseqüentemente, de prevenção do sofrimento psicológico. Ademais, a religiosidade foi indicada como fonte de apoio a mães 61 e a gestantes portadoras de HIV/AIDS 31. Como fonte de interpretação para os acontecimentos da vida, a religiosidade pode representar apoio para o enfrentamento das dificuldades e para a mudança de atitudes.

Apesar desses avanços sociais e dos fatores de proteção mencionados na literatura, encontram- se ainda diversos estudos salientando o quanto a vida social das pessoas portadoras de HIV/AIDS pode ficar prejudicada. Isso se dá principalmente pela presença do preconceito e da estigmatização 31,61,62,63,64. Mesmo com o passar dos anos e com as transformações nas características da epidemia, ainda é forte o impacto social negativo gerado pelo HIV. Os portadores do vírus seguem sendo uma população estigmatizada, vivenciando diversos tipos de preconceito 31,62,63. Conceitualmente, o estigma é atribuído pela sociedade àquele que apresenta um "desvio", ou uma diferença indesejável 64 . Socialmente, dentre outras conotações, a AIDS tem sido entendida como punição a comportamentos imorais, despertando preconceito. Nessa medida, em alguns casos, enfrentar a estigmatização e o preconceito pode parecer mais difícil do que enfrentar a infecção do ponto de vista de saúde física 63 .

Mulheres portadoras de HIV/AIDS têm mencionado que temem e vivenciam preconceito 65 . Com isso, pode surgir a dificuldade de colocação profissional 62 , além dos problemas familiares 66 e daqueles ligados à maternidade 31,65,67,68,69.

Todos esses aspectos constituem o que vem sendo descrito como a morte civil e social dos soropositivos 57,63 . Essa morte simbólica é vivida pelo indivíduo a partir da redução de seus direitos como cidadão, com dificuldades no trabalho, desemprego, discriminação, isolamento, entre outros fatores 45

Como pode ser visto, os sistemas de apoio social podem ser bastante protetores aos indivíduos portadores de HIV/AIDS. Reforça-se nisso a importância de esses sistemas auxiliarem na dissolução do preconceito e da discriminação. Em particular, destaca-se a importância da rede de saúde disponível a essas pessoas, com o oferecimento de diagnóstico, tratamento e prevenção primária, secundária e terciária, que contribuem para a promoção de saúde desses pacientes. Além disso, ressalta-se o papel das ONGs, da legislação brasileira e ainda da religiosidade nesse processo.

\section{Considerações finais}

O presente artigo teve como objetivo examinar a relevância do conceito de resiliência no contexto da infecção pelo HIV/AIDS, com destaque para os possíveis fatores de proteção, os quais, nos estudos revisados, revelaram-se importantes, contribuindo para a saúde geral e para o bemestar das pessoas portadoras de HIV/AIDS. Entre eles, destacam-se o enfrentamento cognitivo e a aceitação da infecção; a participação da família no tratamento e como fonte de apoio afetivo; o 
papel das organizações governamentais e nãogovernamentais e a religiosidade. Isso sugere que indivíduos soropositivos para HIV podem superar a adversidade trazida pela infecção com uma boa qualidade de vida, e esse bom desempenho num contexto extremamente difícil pode ser, então, característico de pessoas resilientes.

Espera-se que o presente estudo ofereça uma primeira sistematização do conceito de resiliência e sua articulação com a situação de infecção pelo HIV/AIDS. Os fatores de proteção apresentados ao longo do artigo não devem ser tomados de forma descontextualizada, e, sim, com base em uma análise que considere cada pessoa nas suas características individuais e na sua rede de relações sociais e afetivas. Além disso, esses fatores de proteção devem ser vistos sempre na relação com os fatores de risco que engendram a situação de adversidade a ser superada.

Tendo em vista a definição de resiliência como a "capacidade do ser humano de superar adversidades", esta deve ser uma compreensão essencial a ser incorporada no entendimento da infecção e tratamento de pacientes com HIV/AIDS. Nesse contexto, é fundamental a participação de todos os profissionais de saúde na promoção de fatores de proteção e de resiliência, considerando o papel de extrema importância desses profissionais para os portadores dessa infecção. A concepção de resiliência contribui para acabar com o estigma e preconceito em relação à doença e desmistifica a questão de que bem-estar e qualidade de vida são estados contraditórios e incongruentes com a vida das pessoas infectadas. Em adição, oferece uma nova perspectiva no campo da prevenção (primária, secundária ou terciária) ao focar no reforço das competências humanas. Esta nova visão, de acordo com Morais \& Koller 19, resgata uma concepção de ser humano como alguém que busca a sua felicidade, bem-estar e saúde, tendo todo potencial para desenvolver estratégias para conseguir esse objetivo. A situação de infecção pelo HIV/AIDS pode ser de extrema adversidade, mas pode ser ressignificada e até vivida como fonte potencial para a mudança e para novas oportunidades diante da vida. Por fim, salienta-se que a promoção de resiliência, além de ser importante no contexto de HIV/AIDS, como buscou demonstrar o presente artigo, é de grande importância em outros contextos, a fim de que se promova a saúde integral das pessoas.

\section{Resumo}

Este artigo busca, com base na revisão da literatura, articular o conceito de resiliência com questões da realidade de vida de pessoas portadoras de HIVIAIDS. Em especial, será enfatizada a análise dos fatores de proteção tradicionalmente descritos como promotores de resiliência (características individuais e rede de apoio social e afetiva). Os estudos revisados revelam que existem importantes fatores de proteção, que contribuem para a saúde e bem-estar dos portadores de HIVIAIDS, entre eles o enfrentamento cognitivo e a aceitação da infecção; a participação da família no tratamento e como fonte de apoio afetivo; o papel das organizações governamentais e não-governamentais e a religiosidade. Acredita-se que a compreensão da resiliência como uma "capacidade do ser humano de superar adversidades" é essencial ao entendimento da infecção e tratamento de pacientes com HIVIAIDS. Isso contribui para acabar com o estigma e preconceito em relação à doença e aos seus portadores. Essa perspectiva desmistifica a questão de que bem-estar e qualidade de vida são estados contraditórios à vida das pessoas infectadas, além de contribuir para a elaboração de novas perspectivas de prevenção e tratamento da infecção por HIVIAIDS.

HIV; Sindrome de Imunodeficiência Adquirida; Literatura de Revisão 


\section{Colaboradores}

As autoras F. T. Carvalho e N. A. Morais fizeram, conjuntamente, o levantamento de literatura e a escrita da versão inicial do artigo. Os autores S. H. Koller e C. A Piccinini fizeram revisões sistemáticas do texto, para a construção da versão final.

\section{Referências}

1. World Health Organization. Global situation of the HIV/AIDS pandemic, end 2003. Weekly Epidemiological Record 2003; 49:417-24.

2. Ramos H, Hinrichsen SL, Corrêa PR, Sá PV, Toscano AC. Infecção pelo HTLV-I/II. Ars Cvrandi 1997; 30:54-61.

3. Ministério da Saúde. Manual de controle das DST. Brasília: Ministério da Saúde; 1999.

4. Brito AM, Castilho EA, Szwarcwald CL. AIDS e infecção pelo HIV no Brasil: uma epidemia multifacetada. Rev Soc Bras Med Trop 2001; 34:207-24.

5. Lopes VGS. HIV - perfil da atual transmissão heterossexual no Brasil. DST - J Bras Doenças Sex Transm 1998;10:41-3.

6. Oliveira DLLC, Meyer DE, Santos LHS, Wilhelms DM. A negociação do sexo seguro na TV: discursos de gênero nas falas de agentes comunitárias de saúde do Programa Saúde da Família de Porto Alegre, Rio Grande do Sul, Brasil. Cad Saúde Pública 2004; 20:1309-18.

7. Ministério da Saúde. Parto, aborto e puerpério: assistência humanizada à mulher. Brasília: Ministério da Saúde; 2001.

8. Sociedade Brasileira de Infectologia. Tratamento hoje. Boletim Terapêutico de HIV/AIDS, DST e Hepatites Virais 2003; 1(4).
9. Siegel K, Lekas HM. AIDS as a chronic illness: psychosocial implications. AIDS 2002; 16:69-76.

10. Masten AS, Garmezy N. Risk, vulnerability and protective factors in developmental psychopathology. In: Lahey BB, Kazdin AE, editors. Advances in clinical child psychology. New York: Plenum Press; 1985. p. 1-52.

11. Poletto M, Wagner TMC, Koller SH. Resiliência e desenvolvimento infantil de crianças que cuidam de crianças: uma visão em perspectiva. Psicol Teor Pesqui 2004; 20:241-50.

12. Yunes MAM, Szymansky H. Resiliência: noção, conceitos afins e considerações críticas. In: Tavares J, organizador. Resiliência e educação. São Paulo: Cortez Editora; 2001. p. 13-42.

13. Rutter M. Stress, coping and development: some issues and some questions. J Child Psychol Psychiatry 1981; 22:323-56.

14. Rutter M. Resilience in the face of adversity: protective factors and resistance to psychiatric disorder. Br J Psychiatry 1985; 147:598-611.

15. Rutter M. Psychosocial resilience and protective mechanisms. Am J Orthopsychiatry 1987; 57: 316-31.

16. Rutter M. Resilience: some conceptual considerations. J Adolesc Health 1993; 14:626-31. 
17. Rutter M. Psychosocial resilience and protective mechanisms. In: Rolf J, Masten AS, Cicchetti D, Nuechterlein KH, Weintraub S, editors. Risk and protective factors in the development of psychopathology. New York: Cambridge University Press; 1996. p. 181-214.

18. Junqueira MFPS, Deslandes SF. Resiliência e maus tratos à criança. Cad Saúde Pública 2003; 19:22735.

19. Morais NA, Koller SH. Abordagem ecológica do desenvolvimento humano, psicologia positiva e resiliência: a ênfase na saúde. In: Koller SH, editor. Ecologia do desenvolvimento humano: pesquisa e intervenção no Brasil. São Paulo: Casa do Psicólogo; 2004. p. 91-107.

20. Cecconello AM. Resiliência e vulnerabilidade em famílias em situação de risco [Tese de Doutorado]. Porto Alegre: Universidade Federal do Rio Grande do Sul; 2003.

21. Hutz CS, Koller SH, Bandeira DR. Resiliência e vulnerabilidade em crianças em situação de risco. In: Koller SH, editor. Aplicações da psicologia na melhoria da qualidade de vida. Porto Alegre: Associação Nacional de Pesquisa e Pós Graduação em Psicologia; 1996. p. 79-86. (Coletâneas da ANPEPP).

22. Yunes MAM. Psicologia positiva e resiliência: o foco no indivíduo e na família. Psicol Estud 2003; 8:75-84.

23. Tavares J. Resiliência e educação. São Paulo: Cortez Editora; 2001.

24. Cowan PA, Cowan CP, Schulz MS. Thinking about risk and resilience in families. In: Hetherington EM, Blechman EA, editors. Stress, coping and resiliency in children and families. New Jersey: Lawrence Erlbaum; 1996. p. 1-38.

25. Morais NA. Um estudo sobre a saúde de adolescentes em situação de rua: o ponto de vista dos adolescentes, profissionais de saúde e educadores [Dissertação de Mestrado]. Porto Alegre: Universidade Federal do Rio Grande do Sul; 2005.

26. Cowen EL, Wyman PA. Resilience in children: the nature and the importance of the concept. Psicol Esc Educ 1998; 2:247-56.

27. Giacomoni C. Bem-estar subjetivo infantil: conceito de felicidade e construção de instrumentos para avaliação [Tese de Doutorado]. Porto Alegre: Universidade Federal do Rio Grande do Sul; 2002.

28. Seligman MEP, Csikszentmihalyi M. Positive psychology: an introduction. Am Psychol 2000; 55: 5-14.

29. Sheldon KM, King L. Why positive psychology is necessary? Am Psychol 2001; 56:216-33.

30. Robinson JL. Are there implications for prevention research from studies of resilience? Child Dev 2000; 71:570-2.

31. Carvalho FT. Maternidade em situação de infecção pelo HIV: um estudo sobre os sentimentos de gestantes [Dissertação de Mestrado]. Porto Alegre: Universidade Federal do Rio Grande do Sul; 2005.

32. Ecknrode J, Gore S. Context and process in research on risk and resilience. In: Garmezy N, Haggerty RJ, Rutter M, Sherrod L, editors. Stress, risk and resilience in children and adolescents. Cambridge: Cambridge University Press; 1996. p. 19-63.
33. Dutra R, Forehand R, Armisted L, Brody G, Morse E, Morse PS, et al. Child resiliency in inner-city families affected by HIV: the role of family variables. Behav Res Ther 2000; 38:471-86.

34. Pivnick A, Villegas N. Resilience and risk: childhood and uncertainty in the AIDS epidemic. Cult Med Psychiatry 2000; 24:101-36.

35. Tangenberg KM. Surviving two diseases: addiction, recovery and spirituality among mothers living with disease. Fam Soc 2002; 82:517-24.

36. Simoni JM, Demas P, Mason HRC, Drossman JA, Davis ML. HIV disclosure among women of African descent: associations with coping, social support and psychological adaptation. AIDS Behav 2000; 4:147-58.

37. Nichols S, Mahoney EM, Sirois PA, Bordeaux JD, Stehbens JA, Loveland KA, et al. HIV-associated changes in adaptive, emotional and behavioral functioning in children and adolescents with hemophilia: results from hemophilia growth and development study. J Pediatr Psychol 2000; 25: 545-56.

38. Turner SG. Resilience and social work practice: three case studies. Fam Soc 2001; 82:441-8.

39. Au A, Chan I, Li P, Chung R, Po LM, Yu P. Stress and health-related quality of life among HIV-infected persons in Hong Kong. AIDS Behav 2004; 8:119-29.

40. Ulla S, Remor EA. Psiconeuroimunologia e infecção por HIV: realidade ou ficção? Psicol Reflex Crít 2002; 15:113-9.

41. Weaver KE, Antoni MH, Lechner SC, Durán REF, Penedo F, Fernandez MI, et al. Perceived stress mediates the effects of coping on the quality of life of HIV-positive women on highly active antiretroviral therapy. AIDS Behav 2004; 8:175-83.

42. Prado G, Feaster DJ, Schwartz SJ, Pratt IA, Smith L, Szapocznik J. Religious involvement, coping, social support and psychological distress in HIV-soropositive African American mothers. AIDS Behav 2004; 8:221-3.

43. Reynolds NR, Testa MA, Marc LG, Chesney MA, Neidig JL, Smith SR, et al. Factors influencing medication adherence beliefs and self-efficacy in persons naive to antiretroviral therapy: a multi-center, cross-sectional study. AIDS Behav 2004; 8:141-50.

44. Ferraz AF. Tornar-se soropositivo: da perplexidade ao confronto com o incógnito. In: Morano L, Guimarães R, organizadores. AIDS: olhares plurais: ensaios esparsos sobre a epidemia de HIV/AIDS. Belo Horizonte: Coordenadoria Estadual de DST/ AIDS de Minas Gerais; 1998. p. 111-40.

45. Seffner F. AIDS, estigma e corpo. In: Ferreira J, editor. Semiologia do corpo. Porto Alegre: Editora da Universidade Federal do Rio Grande do Sul; 1995. p. 391-415.

46. Remor EA. Abordagem psicológica da AIDS através do enfoque cognitivo-comportamental. Psicol Reflex Crít 1999; 12:89-106.

47. Knauth DR. Um problema de família: a percepção da AIDS entre mulheres soropositivas. In: Ferreira J, editor. Semiologia do corpo. Porto Alegre: Editora da Universidade Federal do Rio Grande do Sul; 1995. p. 379-90.

48. Regatto VC, Assmar EML. A AIDS de nossos dias: quem é o responsável? Estud Psicol (Natal) 2004; 9:167-75. 
49. Gulinelli A, Aisawa RK, Konno SN, Morinaga CV, Costardi WL, Antonio RO, et al. Desejo de informação e participação nas decisões terapêuticas em caso de doenças graves em pacientes atendidos em um hospital universitário. Rev Assoc Méd Bras 2004; 50:41-7.

50. Kalichman SC, DiMarco M, Austin J, Luke W, DiFonzo K. Stress, social support, and HIV-status disclosure to family and friends among HIV-positive men and women. J Behav Med 2003; 26:31532.

51. Mellins CA, Ehrhardt AA, Rapkin B, Havens JF. Psychosocial factors associated with adaptation in HIV-infected mothers. AIDS Behav 2000; 4:317-28.

52. Remor EA. Aspectos psicossociais na era dos novos tratamentos da AIDS. Psicol Teor Pesqui 2002; 18:283-7.

53. Serovich JM, Kimberly J, Mosack K, Lewis T. The role of family and friend social support in reducing emotional distress among HIV Positive women. AIDS Care 2001; 13:339-45.

54. Silveira VL, Drachler ML, Leite JC, Pinheiro CA. Characteristics of HIV antiretroviral regimen and treatment adherence. Braz J Infect Dis 2003; 7:194201.

55. Ministério da Saúde. Parto, aborto e puerpério: assistência humanizada à mulher. Brasília: Ministério da Saúde; 2001.

56. Figueiredo RM, Sinkoc VM, Tomazim CC, Gallani MCBJ, Colombrini MRC. Adesão de pacientes com AIDS ao tratamento com anti-retrovirais: dificuldades relatadas e proposição de medidas atenuantes em um hospital escola. Rev Latinoam Enferm 2001; 9:50-5.

57. Figueiredo MAC, Fioroni LN. Uma análise de conteúdo de crenças relacionadas com AIDS entre participantes em O.N.G.S. Estud Psicol (Natal) 1997; 2:28-41.

58. Ministério da Saúde. Assistência a parturientes/ puérperas portadoras do HIV e/ou com sífilis, e seus bebês, nas maternidades. Brasília: Ministério da Saúde; 2002.
59. Joint United Nations Programme on HIV/AIDS. HIV e AIDS nas Américas: uma epidemia multifacetada: Organização Pan-Americana da Saúde: 2001.

60. Valle CG. Identidades, doença e organização social: um estudo das pessoas vivendo com HIV e AIDS. Horizontes Antropológicos 2002; 8:179-210.

61. Siegel K, Schrimshaw EW. Reasons and justifications for considering pregnancy among women living with HIV/AIDS. Psychol Women Q 2001; 25:112-23.

62. Santos NJS, Buchalla CM, Fillipe EV, Bugamelli L, Garcia S, Paiva V. Mulheres HIV positivas, sexualidade e reprodução. Rev Saúde Pública 2002; 36:1223.

63. Guimarães R. A inter-relação estigma e trajetória da doença causada pelo HIV. In: Morano L, Guimarães R, organizadores. AIDS: olhares plurais: ensaios esparsos sobre a epidemia de HIV/AIDS. Belo Horizonte: Coordenadoria Estadual de DST/AIDS de Minas Gerais; 1998. p. 85-110.

64. Parker R, Aggleton P. Estigma, discriminação e AIDS. Rio de Janeiro: Associação Brasileira Interdisciplinar de AIDS; 2001.

65. Paiva V, Lima TN, Santos N, Ventura-Filipe E, Segurado A. Sem direito de amar? A vontade de ter filhos entre homens e mulheres vivendo com HIV. Psicol USP 2002; 13:105-33.

66. D'Cruz P. Engulfing darkness: the impact of HIV/AIDS on the family. Fam Soc 2002; 83:416-30.

67. Silva PA. A (in)certeza da vida: representações sociais de gestantes soropositivas ao HIV/AIDS sobre o nascimento de seu filho(a) [Dissertação de Mestrado]. Rio de Janeiro: Universidade do Rio de Janeiro; 2003.

68. Nelms TP. Burden: the phenomenon of the mothering with HIV. J Assoc Nurses AIDS Care 2005; 16:3-13.

69. Ingram D, Hutchinson SA. Double binds and the reproductive and mothering experiences of HIVpositive women. Qual Health Res 2000; 10:117-32.

Recebido em 13/Jul/2006

Versão final reapresentada em 02/Mar/2007 Aprovado em 19/Abr/2007 
